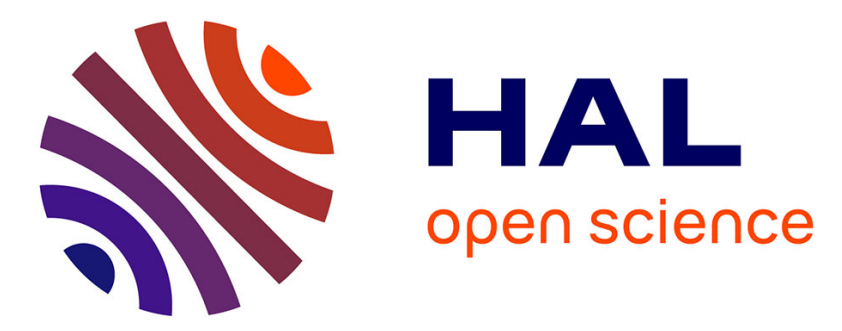

\title{
Numerical study of the variations of magnetic resonance signals caused by surface slope
}

Jean-François Girard, A. Legchenko, Marie Boucher, Jean-Michel Baltassat

\section{To cite this version:}

Jean-François Girard, A. Legchenko, Marie Boucher, Jean-Michel Baltassat. Numerical study of the variations of magnetic resonance signals caused by surface slope. Journal of Applied Geophysics, 2008, 66 (3-4), pp.94-103. 10.1016/j.jappgeo.2008.04.003 . insu-00357012

\section{HAL Id: insu-00357012 \\ https://hal-insu.archives-ouvertes.fr/insu-00357012}

Submitted on 3 Feb 2009

HAL is a multi-disciplinary open access archive for the deposit and dissemination of scientific research documents, whether they are published or not. The documents may come from teaching and research institutions in France or abroad, or from public or private research centers.
L'archive ouverte pluridisciplinaire HAL, est destinée au dépôt et à la diffusion de documents scientifiques de niveau recherche, publiés ou non, émanant des établissements d'enseignement et de recherche français ou étrangers, des laboratoires publics ou privés. 


\title{
Numerical study of the variations of magnetic resonance signals caused by surface slope
}

\author{
J.-F. Girard ${ }^{\mathrm{a}}$, A. Legchenko ${ }^{\mathrm{b}, 1}$,, M. Boucher ${ }^{\mathrm{a}, \mathrm{c},}$ and J.-M. Baltassat ${ }^{\mathrm{a}}$ \\ ${ }^{a}$ Bureau de Recherches Géologiques et Minières (BRGM), 3, avenue C. Guillemin, BP 36009, \\ 45060, Orléans Cedex 2, France \\ ${ }^{\mathrm{b}}$ Institut de Recherche pour le Développement (IRD-LTHE), LTHE, BP53, 38041, \\ GRENOBLE Cedex 9, France
}

'Institut des Sciences de la Terre d'Orléans (ISTO), UMR6113 CNRS/Université d'Orléans, Bâtiment Géosciences, Rue de Saint Amand, BP 6759, 45067 Orléans Cedex 2, France

\begin{abstract}
When performing forward modelling and inversion of Magnetic Resonance Sounding (MRS) data, the water-content distribution is typically assumed to be horizontal (1D case). This assumption is fully justified because MRS is often used for characterizing continuous aquifers in a nearly flat environment. However, MRS can also be used in areas with sharp topographical variations. Following a review of the standard MRS equations when using a coincident transmitter/receiver loop, the mathematical terms potentially affected by tilting of the loop are discussed. We present the results of a numerical modelling exercise, studying a case where the surface is not horizontal and the loop cannot be considered to be parallel to the top of the aquifer. This shows that maximum variations in the MRS-signal amplitude are caused mainly by north- or south-dipping slopes. Slope effects depend on the loop size (a larger loop produces a larger error) especially in the presence of shallow water. With a geomagnetic-field inclination of $65^{\circ}$ and a slope angle $\leq 10^{\circ}$, the topography causes a maximum variation in amplitude of less than $10 \%$. Near magnetic poles and equator, the slope effect is lower and undetectable in most cases. It was found that within a $10 \%$ range of variation in the amplitude, errors introduced into inversions are within the typical uncertainty for MRS inversion and hence no topographic corrections are necessary. Thus, a significant effect from non-horizontal topography might be expected only when data uncertainty is lower than the slope effect (the slope effect is lower than equivalence when data quality is poor). Today, most field data sets are inverted using the modulus of the MRS signal, but some new developments consider the complex signal (both modulus and phase). However, inversion of complex MRS signals, which would provide a higher sensitivity to groundwater distribution, may be affected by slope effect. Thus, the slope orientation and dip angle should be accurately measured in the field when the phase of MRS signals is inverted too.
\end{abstract}

Keywords: MRS; SNMR; Magnetic resonance sounding; Topography; Groundwater 


\section{Introduction}

The magnetic resonance phenomenon is used for non-invasive investigations of groundwater. Magnetic Resonance Sounding (MRS) is a powerful method for hydrogeological purposes since it provides data on the geometry of an aquifer and an estimation of the storage-related parameters. Several authors ([Legchenko and Shushakov, 1998] and [Weichman et al., 2002]) have studied the performance of MRS using a single loop laid on the ground for transmitting an energizing electromagnetic (EM) field at the Larmor frequency and for receiving the response of the groundwater.

The interpretation of MRS signals was improved by including the effect of electrical conductivity of the ground (Trushkin et al., 1995) and the frequency shift due to geomagneticfield variations (Girard et al., 2005). For practical applications, MRS users should know the conditions where these effects should be taken into account. In this paper we investigate the effect of topography when performing coincident loop MRS measurements.

Standard MRS forward modelling and inversion (Legchenko and Valla, 2002) assume a horizontal loop on the Earth's surface over a horizontal water-content distribution in the subsurface (1D case). This assumption is fully justified because MRS is widely used for the characterization of continuous aquifers, which generally occur in a nearly flat environment. However, MRS can also be applied in areas with steep topography. On a slope, the loop generally is not parallel to the horizontal stratification of the water content usually assumed for computing MRS signal.

A review of the standard MRS equations describes the mathematical terms potentially affected by tilting of the loop. We have developed a specific software for considering the tilting of the loop and thus for studying the effects of the dip and orientation of the surface slope on MRS results. The case of a shallow or a deep aquifer is considered, as well as the use of small and large coincident transmitter-receiver loops.

Because the geomagnetic field is involved in the MRS method, the slope effect may vary not only with the array and groundwater distribution, but also with the geographic position of the MRS. In the first section of the paper, geomagnetic conditions common to France are used, while low- and high-latitude cases are presented afterwards.

The calculation of the errors introduced into the MRS results when neglecting the tilting of the loop under various slope conditions, allows us to answer the questions: "What error is introduced into the determination of aquifer geometry?" and "Is the water-content estimate biased?". More generally, when can topographical variations in the surface be neglected and when not?

\section{Modelling}

A new program was developed for computing the MRS response for a coincident transmitterreceiver loop configuration that takes into account not only the standard parameters (size, geomagnetic-field conditions, electrical conductivity of the soil, pulse range, etc.), but also the slope of the surface (Fig. 1). The numerical study focused on the case where horizontal stratification is assumed for the aquifer, but where the topographic surface on which the coincident loop is located is at a definite angle to the horizontal. 
A parallel can be drawn with an aquifer dipping below a horizontal loop if the geomagneticfield vector is rotated with respect to the slope angle and direction. In case of surface slope, the integration of the MRS signal should not be done parallel to the ground surface but horizontally, which implies that the integration below the surface will be laterally limited. Earlier work discussed the case of a dipping aquifer below a horizontal loop, considering the aquifer as 1D at the loop scale (Legchenko et al., 2002) or as a 2D target (Warsa et al., 2003) using closely spaced soundings.

\subsection{MRS equations}

Basic MRS equations are derived from the integration of Bloch's equations. After transmitting an exciting EM-field (magnetic induction $B_{1}$ ) pulse through the loop, characterized by pulse moment $Q$ in A ms, the initial amplitude $E_{0}$ of the MRS signal (a complex variable, including phase information) is:

$$
E_{0}(Q)=\int_{0}^{\infty} K(Q, z) \cdot w(z) d z
$$

for the standard 1D case using a coincident loop. In this equation, $w(z)$ is the water-content distribution over depth and $K(Q, z)$ corresponds to the MRS response of a thin infinite layer of water at depth $z$ for a pulse moment $Q$. Considering the magnetic field transmitted by the loop is elliptically polarized ([Weichman et al., 2002] and [Valla and Legchenko, 2002]), $K(Q, z)$ is used as a linear filter in the modelling process and is defined as:

$$
K(Q, z)=\frac{\omega_{0} M_{0}}{I_{0}} \iint_{x y} 2 B_{t}^{(-)} e^{i P_{0}} \sin \left(\frac{\gamma}{L_{0}} B_{1}^{(+)} Q\right) d x d y
$$

The Larmor frequency $\left(F_{\mathrm{L}}=\omega_{0} / 2 \pi\right)$ is linked to geomagnetic induction through the gyromagnetic ratio $\gamma: \omega_{0}=\gamma \cdot\left|B_{0}\right|$ (for water-molecule protons, $\gamma=0.2675 \mathrm{rad} / \mathrm{s} / \mathrm{nT}$ ). $M_{0}$ is the nuclear magnetization for protons in water at thermal equilibrium and $M_{0}=3.287 \cdot 10^{-3}\left|B_{0}\right|$ at $293 \mathrm{~K}$. Considering that the magnetic field generated by the transmitted magnetic field $B_{1}$ is complex, we can express the phase shifts due to the electromagnetic shift of the signal generated by volume $\mathrm{d} V$ as caused by the electrical conductivity of rocks:

$$
\varphi_{0}(\mathbf{r})=2 \tan ^{-1}\left(\operatorname{Im}\left(B_{1}\right) / \operatorname{Re}\left(B_{1}\right)\right)
$$

with $\mathbf{r}=r(x, y, z)$ the coordinate vector. $B_{1}{ }^{(+)}(\mathbf{r}, \rho(\mathbf{r}), \alpha$, slope $)$ and $B_{1}{ }^{(-)}(\mathbf{r}, \rho(\mathbf{r}), \alpha$, slope $)$ are respectively the co-rotating and counter-rotating components of the transmitting magnetic field $B_{1}$ perpendicular to the geomagnetic-field $B_{0}$. They depend on the subsurface resistivity $\rho(\mathbf{r})$, on the geomagnetic-field inclination $\alpha$, and on the slope dip and direction.

If the loop is inclined, the exciting magnetic field generated by the loop is tilted as well and the perpendicular component will be modified, thus introducing the so-called topographic effect. This effect is modulated by the geomagnetic-field properties and hence by the geographic position. The magnetic field transmitted by the loop is computed using Anderson's 
approach for calculating the Hankel transforms (Anderson, 1989). This routine allows considering conductive layers parallel to the loop. In our program, the magnetic induction due to the alternating current in the loop is calculated for the horizontal case. After that, the magnetic field transmitted by the loop is rotated with respect to the direction and dip of the slope. One should note that only electrical resistivity variations parallel to the ground can be considered (Fig. 1). The rest of the calculation is similar to the standard MRS horizontal case, but because the linear filter $K(Q, z)$ corresponds to a horizontal stratification of groundwater, the numerical integration is laterally limited to the underground. The exciting field in the air is not computed because it is not used in the MRS-signal calculation (only groundwater contributes to the MRS signal).

\subsection{D assumption}

In hydrogeology, MRS is commonly used for mapping hydrodynamic properties of a catchment, using a coarse grid of several hundreds of metres to several kilometres between stations. This provides valuable information for the understanding of water flow at the catchment scale.

Generally speaking, topographic variation is a 3D issue, but when the slope can be considered as flat at the loop scale the problem remains a 1D case (Legchenko et al., 2006). If the slope is very steep, a shallow aquifer may reach the surface strongly limiting the lateral extent of the aquifer inside or near the loop. This case is clearly a 2D or 3D issue that cannot be answered with only one sounding. In that case, a profile with multiple soundings should be used with overlapping loops (Boucher et al., 2006), or with a separate loop configuration (Hertrich et al., 2005) to obtain an acceptable resolution. A 1D assumption is made for the electrical properties of the soil. The MRS response can be significantly affected by a contrasted 2D structure of electrical resistivity (Braun et al., 2005) but one would need some 2D measurements to take it into account.

This paper studies the slope effect assuming sub-horizontal layered aquifers, which is a regular condition when conducting MRS. We focused our study on the effect topography has on a single sounding when the assumption of a 1D groundwater distribution is fulfilled. We applied a simple geometric constraint for controlling the effectiveness of the 1D assumption (avoiding the clearly 2D/3D case of a laterally limited aquifer):

\section{$Z \geq D \times \sin (P)$}

where $Z$ is the depth from loop centre to the top of aquifer (Fig. 1), $D$ the loop diameter and $P$ the slope angle. This constraint implies that the top of aquifer does not reach the surface inside a concentric 2-D diameter circle within the loop. For a 50-m-diameter loop, the condition of laterally unlimited aquifer (at the loop scale) is fulfilled if $Z>8.7 \mathrm{~m}$ for a $10^{\circ}$ slope, and $Z>25 \mathrm{~m}$ for a steep $30^{\circ}$ slope.

The standard field conditions used in the modelling were kept almost constant. The subsurface was assumed to be a half-space with a $200-\mathrm{Ohm} \mathrm{m}$ resistivity, although a multilayer conductive earth model could have been considered, with subsurface layers of different conductivity parallel to the ground surface. We considered the geomagnetic conditions in central France with a $2001-\mathrm{Hz}$ Larmor frequency and a $65^{\circ}$ geomagnetic-field inclination. 
The surface-slope effects on MRS when varying the geomagnetic inclination, or in a lowresistivity case $(1 \mathrm{Ohm} \mathrm{m})$, were specifically investigated.

\subsection{Calculation of slope effect}

For estimating the slope effect on MRS amplitude, we used an error defined as:

$\mathrm{err}=\max \left(\frac{E_{\text {lopo }}(Q)-E_{\text {flar }}(Q)}{E_{\text {flat }}(Q)}\right) \times 100$

where $Q$ varies from 0 to the maximum pulse value of the sounding curve. Because the slope effect is generally small and not constant along the MRS curve, this indicator is based on the maximum effect along the MRS sounding. When the shape of the full sounding is changed, this effect is discussed in detail.

\section{Results}

\subsection{Variations caused by slope orientation}

Considering a 5-m-thick aquifer at a depth from 10 to $15 \mathrm{~m}$, with a $10 \%$ water content, we calculated the MRS curves with circular 25-m-diameter (2 turns) and 50-m-diameter (1 turn) loops laid on a $10^{\circ}$ dipping surface. We investigated the effect of slope orientation relative to magnetic north. In Fig. 2, the MRS amplitude is shown against the pulse moment for azimuth variations from north to south via east direction.

It can be seen that the largest variations in the MRS signal are caused when the slope dips north or south. Focusing on the first amplitude peak response for the 25-m-loop and compared to the horizontal surface case, the amplitude decreases by $-10 \%$ when the slope dips south and increases by $+5 \%$ when it dips north; for the 50-m-loop, these values are respectively $12 \%$ and $+6 \%$. The phase of MRS signal is not affected during the first peak response, which corresponds to the maximum of aquifer response when proton magnetization vectors reach a $90^{\circ}$ angle with the geomagnetic field. A phase variation, corresponding to the basic MRS response integrated at a constant depth, is observed even for the horizontal case when part of the proton magnetization vectors reaches a $270^{\circ}$ tilting angle after the first peak (Legchenko and Valla, 2002).

For this shallow aquifer, the topographic effect is stronger with the 50-m-diameter loop than with the 25-m-diameter one. In addition, our results show that, with a larger loop, not only the amplitude but also the phase after the first peak of the MRS response changes.

We have shown only half of directions because we observed an east-west symmetry. This symmetry can be easily understood because the active part of transmitted magnetic field is the component perpendicular to the geomagnetic field, and hence the situation is symmetric if the slope dips $P$ degrees east or west. We will focus later on the case where this symmetry disappears because of the elliptical polarization of the loop magnetic field in a low-resistivity 
environment. This symmetric (east-west) and asymmetric (north-south) behaviour is directly linked to the focalization of MRS sensitivity below the loop, caused by the inclination of the geomagnetic field discussed in previous works ([Hertrich et al., 2005] and [Girard et al., 2007]). The effect of this focalization was directly observed on 2D field data experiments above a karst conduit (Boucher et al., 2006).

\subsection{Variations caused by aquifer depth and loop size}

Considering the north-south slope direction, where the slope effect is the largest, we calculated the MRS response for $1^{\circ}$ to $30^{\circ}$ slope angles. Two cases, each with a water content of $10 \%$ were studied: a shallow 5-m-thick aquifer at 10 to $15 \mathrm{~m}$ depth (Fig. 3), and a deeper 10 -m-thick aquifer at a depth from 30 to $40 \mathrm{~m}$ (Fig. 4). Using a small (25 m diameter and 2 turns) loop, the computed MRS amplitude for increasing pulse moments shows variations reaching $\pm 10 \%$ depending on the slope. Phase variations (up to $10^{\circ}$ ) oscillating along the main trend are also observed.

Using the larger 50-m-diameter and 1-turn loop, the response of the shallow aquifer is slightly increased $(+5 \%)$ when dipping north but is clearly decreased $(-15 \%)$ when dipping $10^{\circ}$ south, while the phase variation after the first peak is much larger than that observed with the smaller loop. For deeper aquifers the slope effect is very slight, except when the slope dips $30^{\circ}$ south where the signal is significantly lower $(-20 \%)$ and the first peak maximum is reached for lower pulse moment. It is seen that when the slope dips to the north, even a $30^{\circ}$ slope has a small effect. For larger loops $100 \mathrm{~m}$ and $150 \mathrm{~m}$ diameter (Fig. 4c and d) the deeper penetration depth makes the situation similar to the case of the $50 \mathrm{~m}$ diameter loop response of the shallow aquifer (Fig. 3). As one could have expected, the slope effect variation caused by aquifer depth is scaled by the loop diameter.

\subsection{Variations caused by ground resistivity}

The case presented in Fig. 3, a shallow (10-15 m) aquifer with 10\% water content, was computed in a low-resistivity environment (1 Ohm $\mathrm{m}$ ) for a 50-m loop (Fig. 5). Here, the east-west symmetry vanishes. In a layered electrically conductive medium, the response of the eastern and western half-spaces below the loop do not have the same response, due to the co- and counter-rotative components of the exciting field (Valla and Legchenko, 2002). The east-west asymmetry is clear in Fig. $5 \mathrm{a}$ and $\mathrm{b}$ when the direction of a $10^{\circ}$ slope varies.

In the north-south direction (Fig. 5c), the effect is nearly undetectable until a $5^{\circ}$ slope to the north. As in the previous cases, the strongest slope effect remains in the southerly direction $(-20 \%)$. But, in such a conductive environment, the slope effect always decreases the amplitude, whereas in the same case but with a $200-\mathrm{Ohm} \mathrm{m}$ resistivity (Fig. 3), a north slope slightly increases the MRS amplitude and a south slope decreases and flattens the first peak response.

\subsection{Variations caused by the geomagnetic field}

Because it is directly linked to the geomagnetic field, the slope effect for a low and a high geomagnetic inclination was also investigated. Fig. 6 shows the synthetic results from soundings done on a $10^{\circ}$ slope, and similar aquifer conditions as described earlier in Fig. 2 and Fig. 3, where the geomagnetic inclination is $5^{\circ}$ and a Larmor frequency of $1440 \mathrm{~Hz}$ applies (field conditions in Niger). As expected, signal amplitude is lower because the 
geomagnetic-field magnitude is lower. A comparison with the horizontal case shows small variations that apparently are independent of the slope direction (Fig. 6a and b), which is explained by the geomagnetic field that is sub-horizontal thus causing the disappearance of a north-south asymmetry. So, though MRS measurements at a low magnetic latitude have the disadvantage of low amplitudes, there is almost no slope effect.

The same situation will be found at the poles where the geomagnetic field is sub-vertical (Fig. $7 \mathrm{a})$ with an identical response in all directions. The higher amplitude is due to the corresponding higher amplitude of the geomagnetic field (60 $000 \mathrm{nT}$ corresponding to 2 $245 \mathrm{~Hz}$ Larmor frequency). A $5^{\circ}$ slope is nearly undetectable and a $10^{\circ}$ slope generates a $7 \%$ amplitude variation on the first peak (Fig. 7b). The second peak is almost cancelled compared with the horizontal case.

Our observations of the slope effect on MRS are confirmed by a recent study (Rommel et al., 2006). They studied the case of a dune above homogeneous water content in the ground and showed the maximum effect until a $30^{\circ}$ slope to be less than $2 \%$. Their study is in good agreement with ours topography has a small effect on the coincident loop results. They have moreover shown that slope variation between the TX and RX loops has to be taken into account for separate loop measurements.

\subsection{Errors induced into inversion results}

For the field geophysicist it is important to know how surface topography affects the accuracy of MRS results. Should the slope be carefully measured in the field and taken into account in the inversion process, or is this not necessary? The worst case of a shallow aquifer was chosen to underline the artefacts on a 50-m-diameter loop measurement with $65^{\circ}$ geomagnetic inclination. Three synthetic datasets were produced with slopes dipping $10^{\circ}$ north, east and south for a 5-m-thick aquifer at a depth of 10 to $15 \mathrm{~m}$ and with a $10 \%$ water content. A standard 1D horizontal model was used, without the slope effect, to fit the synthetic dataset (Fig. 8).

When the $10^{\circ}$ slope dips north, south or east, a very good fit is obtained: both amplitude and phase fit in all three cases. For scaling the observed variations, the horizontal-loop case is shown as a dashed line on all graphs in Fig. 8 and Fig. 9. The models in Fig. 8 have some variations with the exact model (Table 1), but the centre of the aquifer layer is nearly unchanged. Because MRS is sensitive to the total number of water molecules, there is a well known trade-off between thickness $\Delta z$ and water content $W$ for an aquifer at the same depth. To overcome this equivalence, a comparison of the product of $\Delta z \cdot W$ was made: this product is $0.50 \mathrm{~m}$ for the north-dipping slope, $0.47 \mathrm{~m}$ for the east-dipping one, and $0.46 \mathrm{~m}$ for the south-dipping slope, whereas the exact model is $0.50 \mathrm{~m}$ ( $5 \mathrm{~m}$ thick with $10 \%$ water content). For each slope orientation, the models provide a robust estimation of $\Delta z \cdot W$.

In parallel, the same modelling was run with a constraint on the aquifer thickness, i.e. $5 \mathrm{~m}$ thick, equal to exact case (Fig. 9). A priori information on the aquifer geometry (from boreholes for instance) can help to decrease the inaccuracy of the water-content estimation due to the equivalence between thickness and water content (i.e. several combinations of thickness and water content may generate the same sounding curve, as it is the case in electrical soundings for different combinations of thickness and resistivity values). 
When the thickness of the aquifer is constrained, i.e. only depth to aquifer and water content are free parameters, the MRS curves for east- and north-dipping slopes when neglecting the topography can be fitted to a satisfying level to MRS amplitude, resulting in a very similar result to that of the exact model (Table 1). However, the phase cannot be fitted. The case of a south-dipping slope $\left(10^{\circ}\right.$ inclination) cannot be accurately fitted on either amplitude or phase. The best fit corresponds to an underestimation of water content $(7.6 \%$ instead of $10 \%)$ and depth $(8 \mathrm{~m}$ instead of $10 \mathrm{~m})$. This is unsatisfactory because these parameters are outside the range of any equivalence with the exact model $\Delta z \cdot W=0.38 \mathrm{~m}$.

Finally, models that fit the complex response can be found if the slope is neglected (Fig. 8), but errors in the aquifer geometry are generated that cannot be resolved by only fixing some parameters (from a geological log or other geophysical measurements). When the complex inversion of the MRS signal will be used, inversion on both the signal amplitude and the signal phase, the slope effect should not be neglected. Nevertheless, when using minimum signal-amplitude error as criterion for inversion (as currently the norm in standard processing), the variations due to the slope effect can be neglected in most cases.

\section{Field data}

A data set was obtained at three elevations along the surface slope of the Pyla sand dune in western France (Vermeersch et al., 2002). The topography along a vertical profile is shown in Fig. 10. The piezometric level is extrapolated from a nearby borehole (Legchenko et al., 2002) and the sea level. The electrical resistivity logs are derived from two positions of timedomain electromagnetic soundings. Taking into account the ground resistivity, a $60-\mathrm{m}$-side square loop, $55^{\circ}$ geomagnetic inclination and a $1963 \mathrm{~Hz}$ Larmor frequency, all three MRS soundings were inverted considering a flat topography and, a $12^{\circ}, 23^{\circ}$ and $16^{\circ}$ slope dipping west, respectively at $10 \mathrm{~m}$ (station A), $25 \mathrm{~m}$ (station B) and $40 \mathrm{~m}$ (station C) above the estimated piezometric level. As observed before on the synthetic cases, a good data fit is obtained whether neglecting the slope or not (Fig. 11a). The fitting error decreases below the noise level, estimated (Girard et al., 2005) for each pulse from a noise recording before each signal measurement. Noise levels are respectively $25 \mathrm{nV}, 13 \mathrm{nV}$ and $15 \mathrm{nV}$ on average for stations A, B and C, and are illustrated by the error bars on the sounding curves (Fig. 11a) equal to twice the noise-amplitude estimation.

Inversion results for station A (Fig. 11b) show a shallow water-saturated zone, whether neglecting the slope or not $\left(12^{\circ}\right.$ for station A). Geological truth is not available, and would be not easy to obtain because of perched water due to capillarity and sea-level variations. For the issue of our methodological study, we should focus on the difference between inversion results that are clearly impacted by the surface-slope effect. In this case the loop (60 m side) is large if compared to the water-table depth $(10-20 \mathrm{~m})$. One should note that in this case, the

1D assumption as arbitrarily defined in this study by Eq. (3) is just obtained (because 60 $\sin \left(12^{\circ}\right)=12.5 \mathrm{~m}$ ), and that the situation could be considered as a $2 \mathrm{D}$ issue. So, the result including the surface slope is certainly more reliable, which implies that in such a situation the surface slope should be precisely measured in the field to obtain a reliable data inversion. For stations B and C (Fig. 11c and d), the water content is decreased if the slope is neglected, in agreement with the observation on synthetic in Fig. 2b, but the estimation of the watercontent distribution with depth is little affected. 
Because of the lack of information about the real water content inside the dune, it is not possible to fix some parameters to reduce the equivalence in inversion results (as done on synthetic data in Fig. 9) and observe if neglecting the slope may lead to erroneous interpretation. However, in most practical field cases, the solution including the surface-slope effect is within the equivalence due to the data noise.

\section{Conclusions}

The numerical results reveal that, in medium latitudes, maximum variations in MRS amplitude are caused by a slope in the N-S direction. Slope effects depend on the loop size (larger loop $=$ larger error) especially in the presence of shallow aquifers. When the slope angle is $\leq 10^{\circ}$, the topography causes the maximum amplitude to vary by $10 \%$ when the dip is south.

The same case in a conductive environment shows different behaviour: the amplitude decreases in all slope directions and the east-west symmetry disappears as an effect of the elliptically polarized exciting field. A stronger slope effect was numerically estimated for a shallow aquifer (10-15 m), until $-20 \%$ amplitude for a $10^{\circ}$ south-dipping slope.

When MRS is done in areas of low or high geomagnetic inclination, near the magnetic equator or poles, the slope effect is equal in all directions and is lower than at medium latitudes.

Within a range of $10 \%$ variation in the amplitude in the north direction, it was found that errors introduced into inversions fall within the typical uncertainty of MRS field measurements (Girard et al., 2005) and hence the standard 1D horizontal approach provides good results. This inaccuracy is less than the typical error of inversion of real data caused by EM noise. Therefore, effects caused by topography have to be suspected when data quality is very good and the slope is $>10^{\circ}$.

Nevertheless, the inversion of complex MRS signals that would provide a higher sensitivity to groundwater distribution may be affected by slope effect. Thus, slope orientation and dip angles should be measured in the field in the case of complex inversions.

\section{Acknowledgements}

The presented research results were partly funded by the French national research program PRECODD/REMAPRO. Authors are grateful to Iris-Instruments for providing the MRS data of the Pyla sand dune.

\section{References}

Anderson, 1989 W.L. Anderson, A hybrid fast Hankel transform algorithm for electromagnetic modelling, Geophysics 54 (1989), pp. 263-266

Boucher et al., 2006 M. Boucher, J.-F. Girard, A. Legchenko, J.-M. Baltassat, N. Dorfliger and K. Chalikakis, Using 2D inversion of magnetic resonance soundings to locate a waterfilled karst conduit, Journal of Hydrology 330 (2006), pp. 413-421. 
Braun et al., 2005 M. Braun, I. Rommel, M. Hertrich and U. Yaramanci, Modelling of MRS signals over 2D electrically conductive structures, Proceedings of EAGE Near Surface 2005 Meeting, Palermo/Italy (2005).

Girard et al., 2005 J.-F. Girard, A. Legchenko and M. Boucher, Stability of MRS signal and estimating data quality, Near Surface Geophysics 3 (2005), pp. 187-194.

Girard et al., 2007 J.-F. Girard, M. Boucher, A. Legchenko and J.-M. Baltassat, 2D magnetic resonance tomography applied to karstic conduit imaging, Journal of Applied Geophysics 63 (2007), pp. 103-116.

Hertrich et al., 2005 M. Hertrich, M. Braun and U. Yaramanci, Magnetic resonance soundings with separated transmitter and receiver loops, Near Surface Geophysics 3 (2005), pp. 141154.

Legchenko and Shushakov, 1998 A.V. Legchenko and O.A. Shushakov, Inversion of surface NMR data, Geophysics 63 (1998), pp. 75-84.

Legchenko and Valla, 2002 A. Legchenko and P. Valla, A review of the basic principles for proton magnetic resonance sounding measurements, Journal of Applied Geophysics $\mathbf{5 0}$ (2002), pp. 3-19.

Legchenko et al., 2002 A. Legchenko, J.-M. Baltassat, A. Beauce and J. Bernard, Nuclear magnetic resonance as a geophysical tool for hydrologists, Journal of Applied Geophysics $\mathbf{5 0}$ (2002), pp. 21-46.

Legchenko et al., 2006 A. Legchenko, M. Descloitres, A. Bost, L. Ruiz, M. Reddy, J.-F. Girard, M. Sekhar, M. Kumar and J.-J. Braun, Resolution of MRS applied to the characterization of hard-rock aquifers, Ground Water 44 (2006), pp. 547-554.

Rommel et al., 2006 I. Rommel, M. Hertrich and U. Yaramanci, The effect of topography on MRS measurements with separated loops, Proceedings of MRS - 3rd International Workshop 25-27 October 2006, Madrid, France (2006).

Trushkin et al., 1995 D.V. Trushkin, O.A. Shushakov and A.V. Legchenko, Surface NMR applied to an electroconductive medium, Geophysical Prospecting 43 (1995), pp. 623-633.

Valla and Legchenko, 2002 P. Valla and A. Legchenko, One-dimensional modelling for proton magnetic resonance sounding measurements over an electrically conductive, Journal of Applied Geophysics $\mathbf{5 0}$ (2002), pp. 217-229.

Vermeersch et al., 2002 F. Vermeersch, B. Texier and J. Bernard, Comparison of various loop geometries in magnetic resonance soundings on the Pyla sand dune (France), Proceedings of MRS - 2nd International Workshop 19-21 November 2003, Orléans, France (2002).

Warsa et al., 2003 W. Warsa, O. Mohnke and U. Yaramanci, 3D modelling and assessment of 2D inversion of surface NMR, Proceedings of MRS - 2nd International Workshop 19-21 November 2003, Orléans, France (2003). 
Weichman et al., 2002 P.B. Weichman, D.R. Lun, M.H. Ritzwoller and E.M. Lavely, Study of surface nuclear magnetic resonance inverse problems, Journal of Applied Geophysics $\mathbf{5 0}$ (2002), pp. 131-147.

\section{Figures}

a)

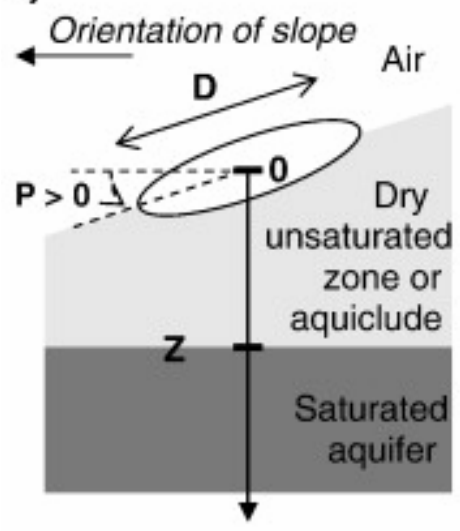

New model with inclined loop above horizontal aquifer b)

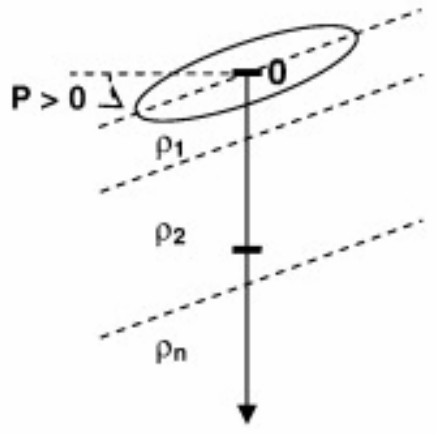

Layered geoelectrical model parallel to the ground

Fig. 1. Vertical section in the steepest slope direction of the new model considering an arbitrary surface slope above horizontally stratified aquifer layers (a) but with resistivity layers parallel to the surface ground (b). 

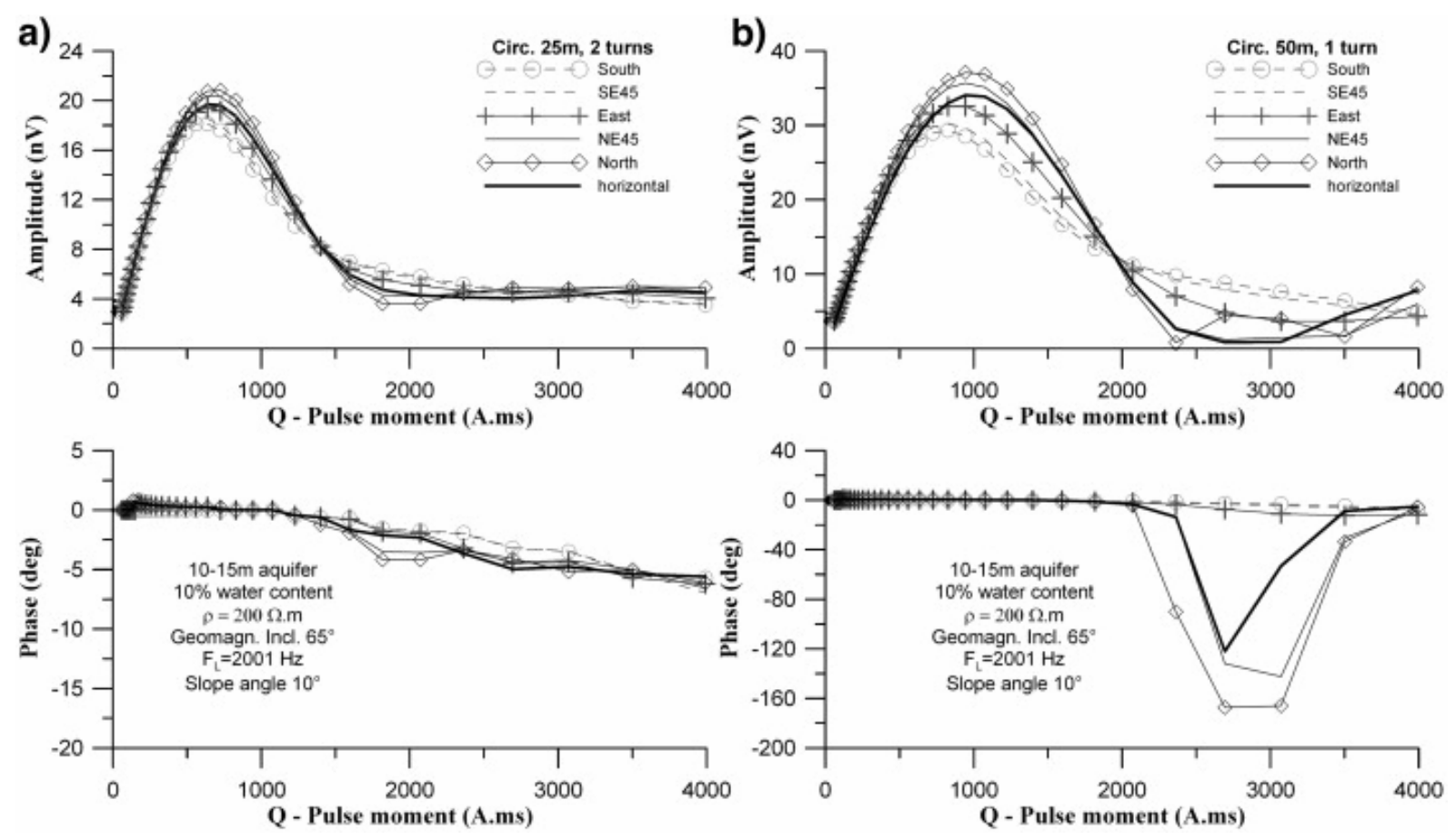

Fig. 2. Effect of a $10^{\circ}$ slope direction on MRS response of a shallow aquifer (10\% water content, 10-15 m) using $25 \mathrm{~m}$ (a) and $50 \mathrm{~m}$ (b) loop diameters. 

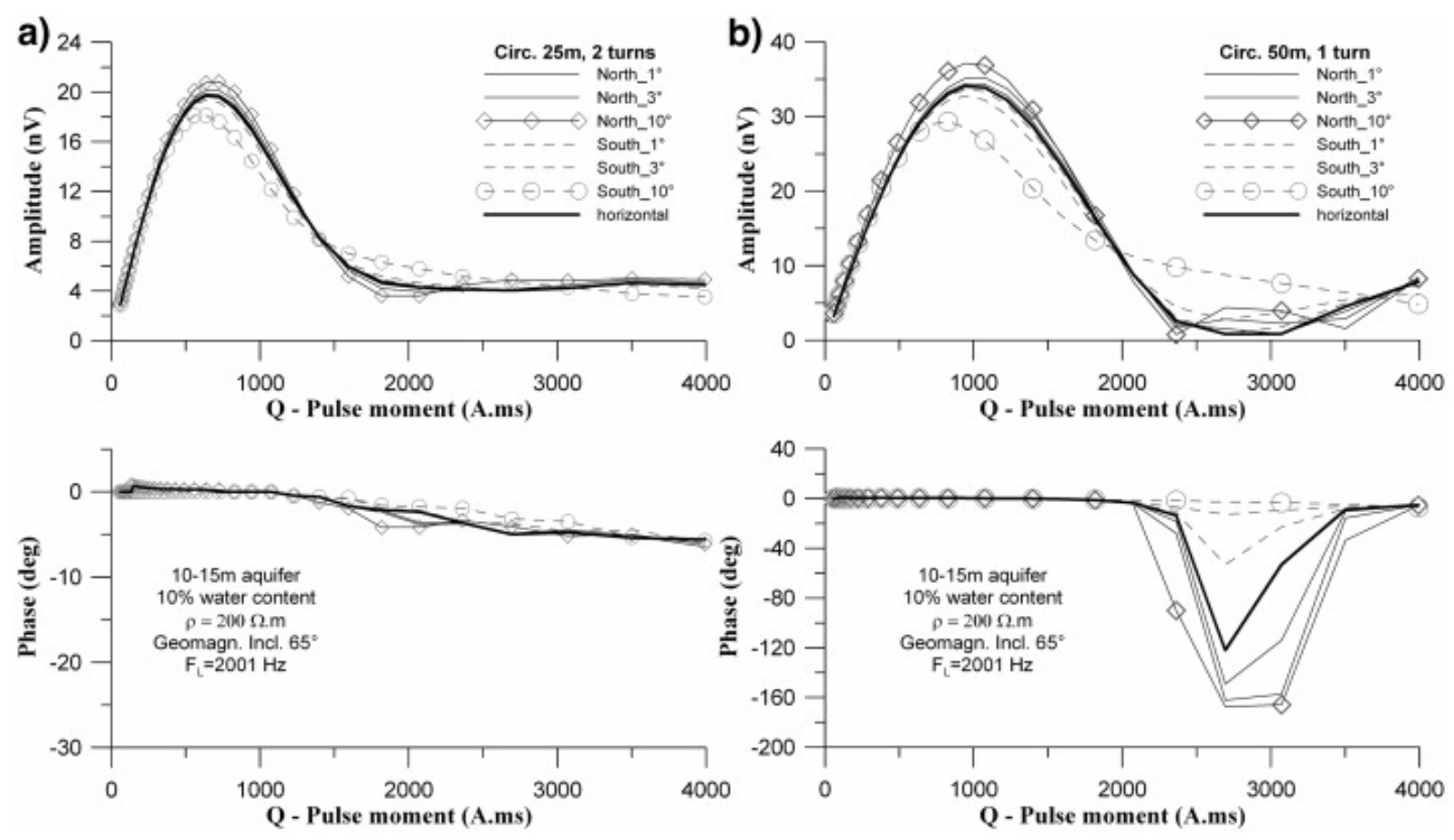

Fig. 3. Slope angle effect, in north-south direction, on MRS response of a shallow aquifer (10\% water content, $10-15 \mathrm{~m}$ ) using $25 \mathrm{~m}$ (a) and $50 \mathrm{~m}$ (b) loop diameters. 
a)
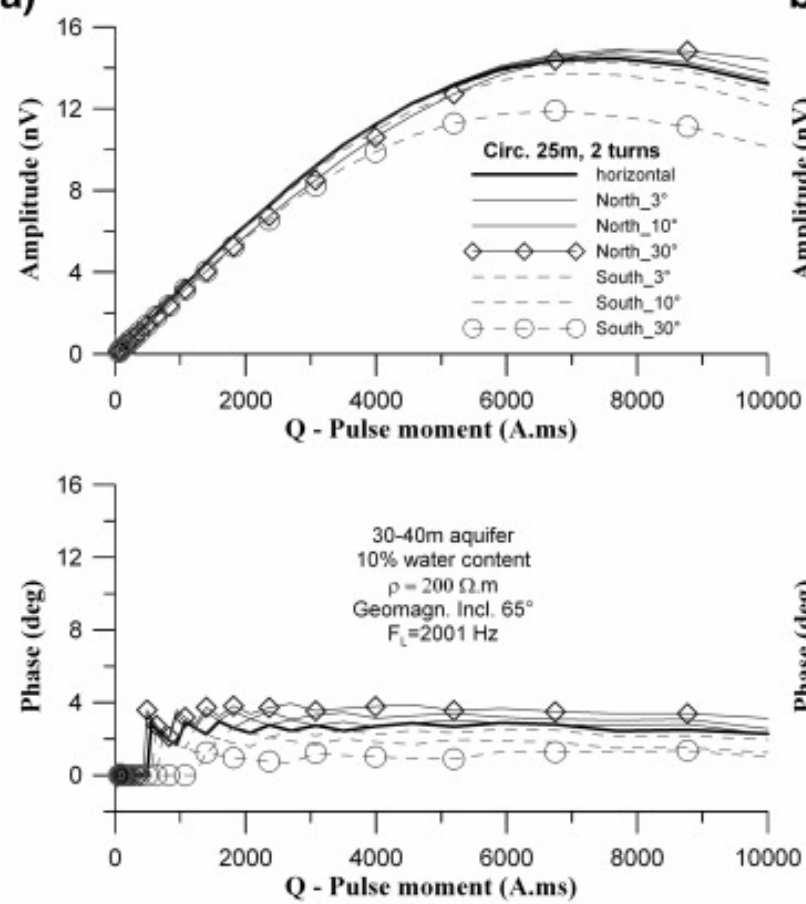

c)
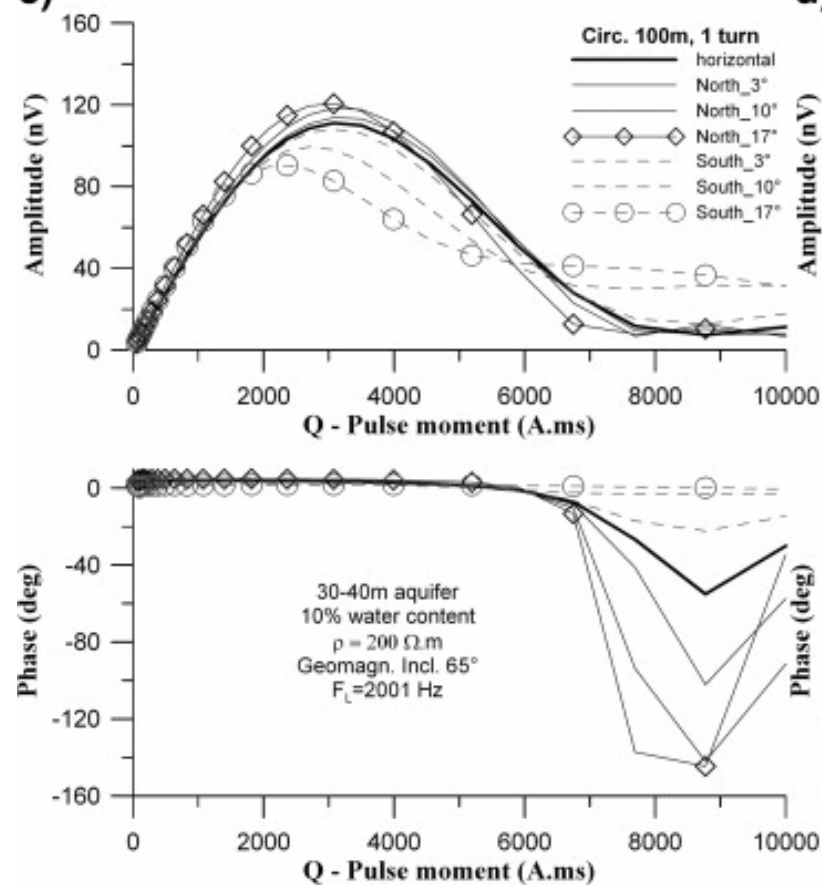

b)
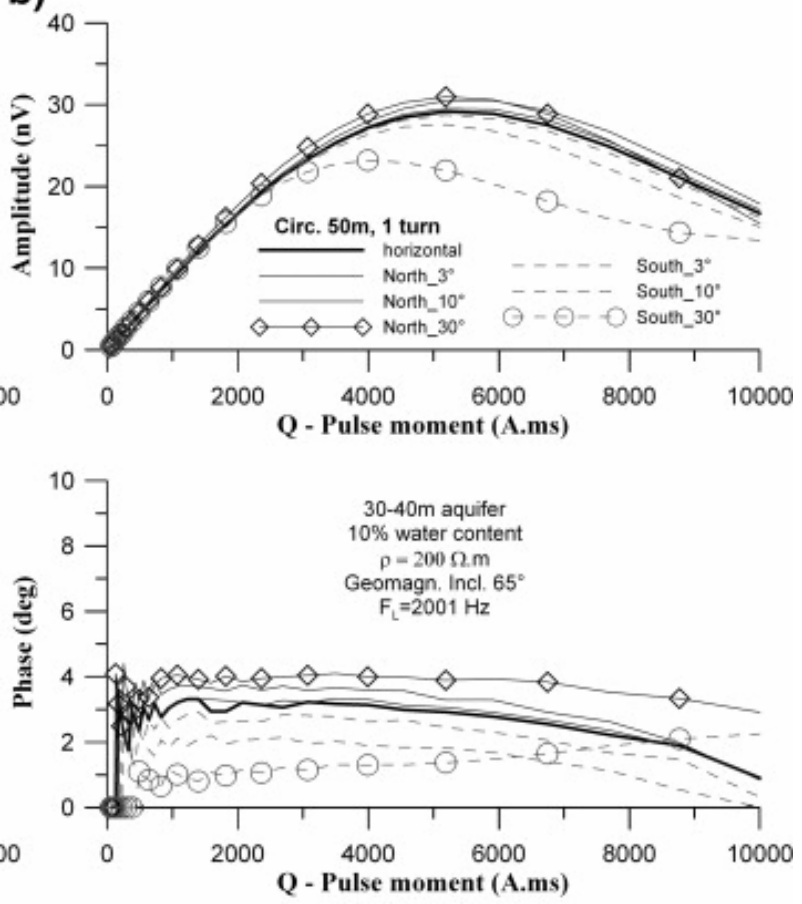

d)
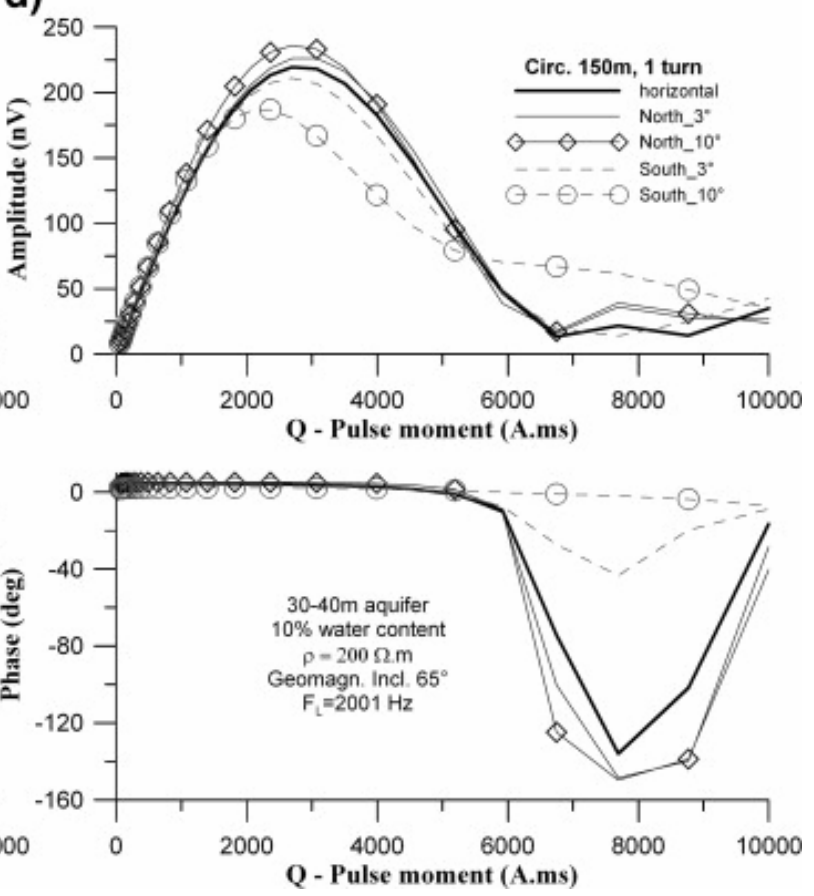

Fig. 4. Slope angle effect, in north-south direction, on MRS response of a deeper aquifer (10\% water content, 30-40 m) using $25 \mathrm{~m}$ (a), $50 \mathrm{~m}$ (b), $100 \mathrm{~m}$ (c) and $150 \mathrm{~m}$ (d) loop diameters. 
a)
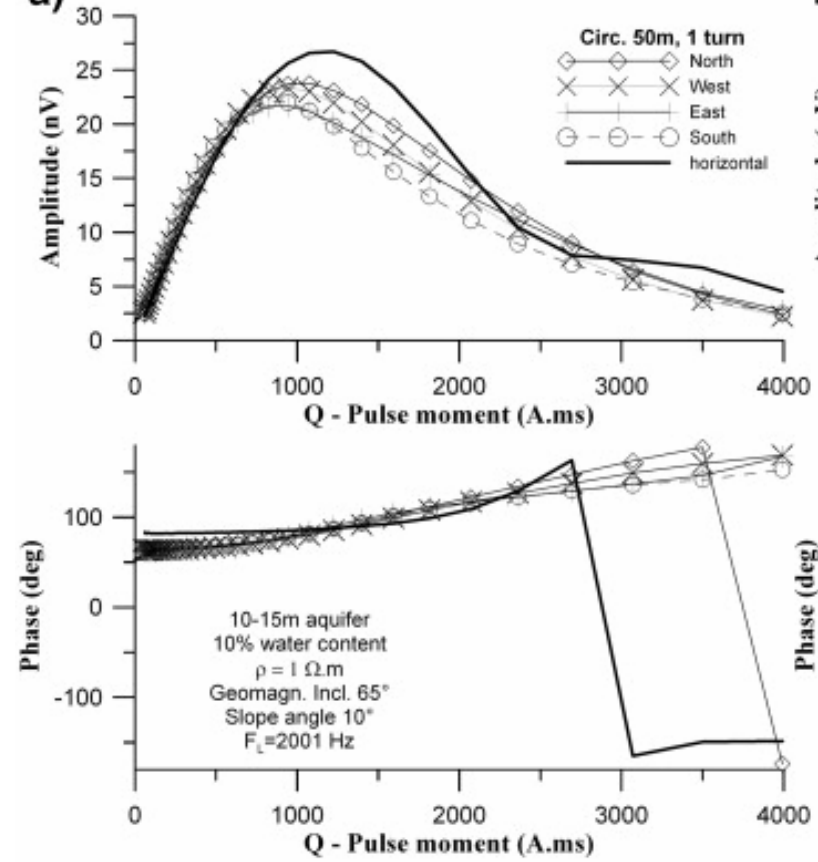

b)
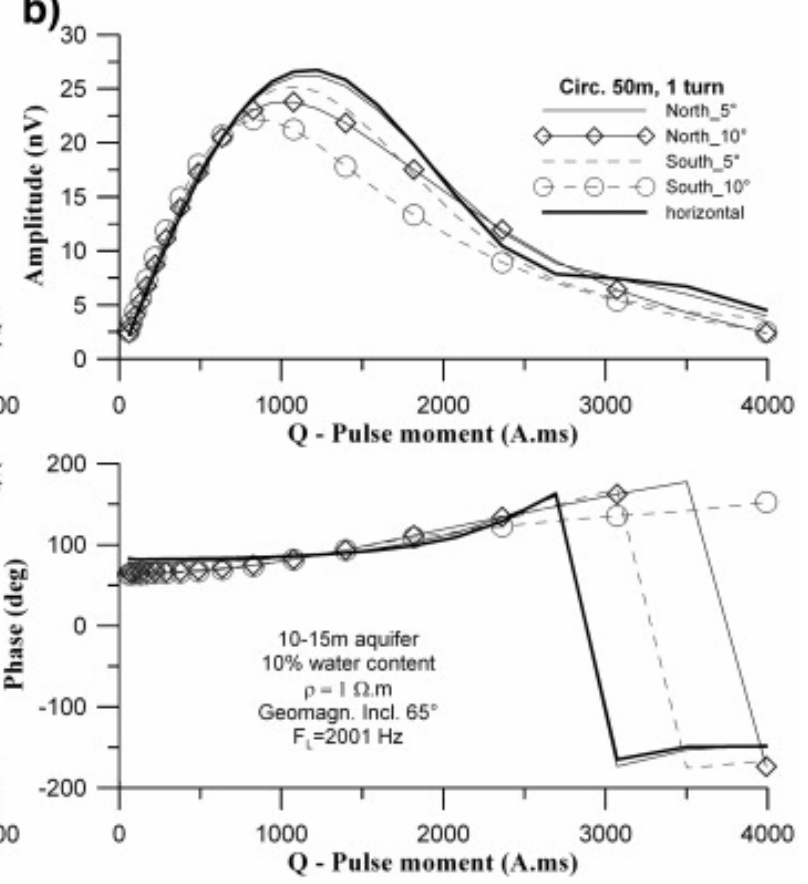

Fig. 5. Effect of a $10^{\circ}$ slope direction (a) and slope angle in north-south direction (b) on MRS response of a shallow aquifer (10\% water content, $10-15 \mathrm{~m})$ using $50 \mathrm{~m}$ loop diameter in a $1 \mathrm{Ohm}$ m medium. 

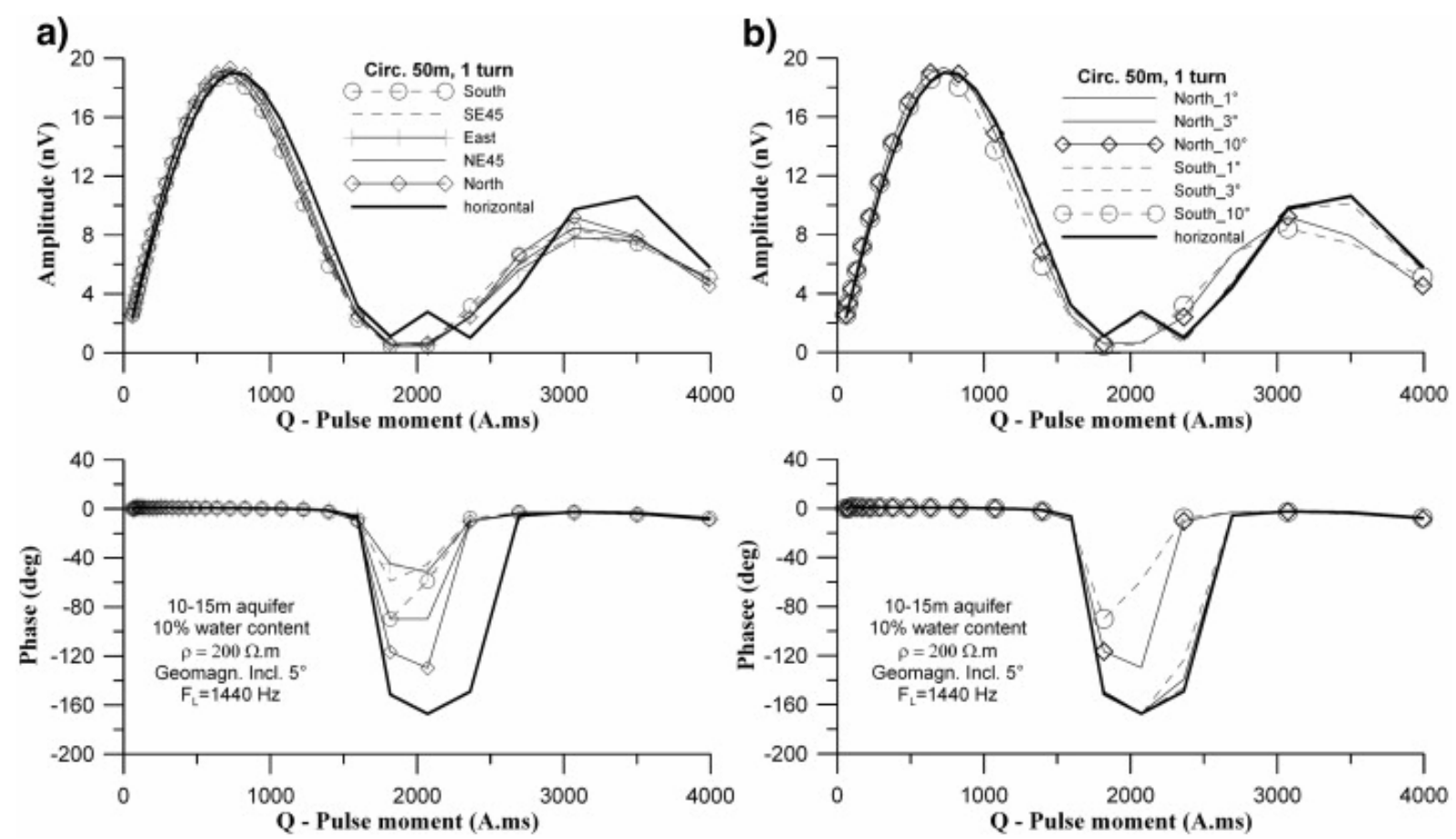

Fig. 6. Effect of a $10^{\circ}$ slope direction (a) and slope angle in north-south direction (b) on MRS response of a shallow aquifer (10\% water content, 10-15 m) using $50 \mathrm{~m}$ loop diameter with $5^{\circ}$ geomagnetic inclination (Niger). 
a)
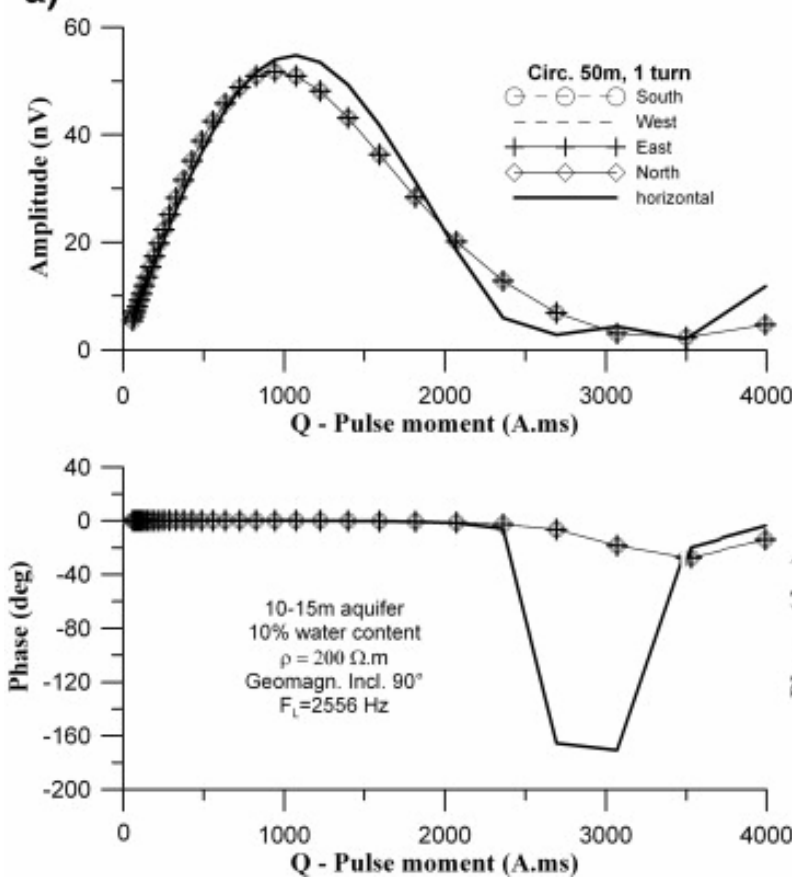

b)
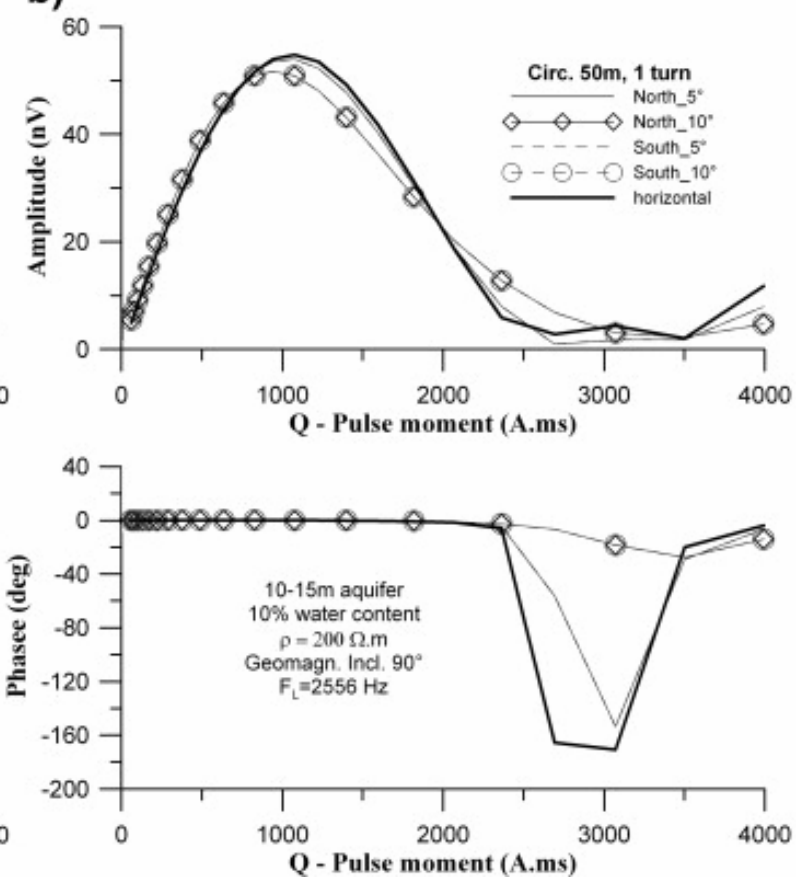

Fig. 7. Effect of a $10^{\circ}$ slope direction (a) and slope angle in north-south direction (b) on MRS response of a shallow aquifer (10\% water content, $10-15 \mathrm{~m})$ using $50 \mathrm{~m}$ loop diameter at the pole (90 geomagnetic inclination, $60000 \mathrm{nT}$ ). 

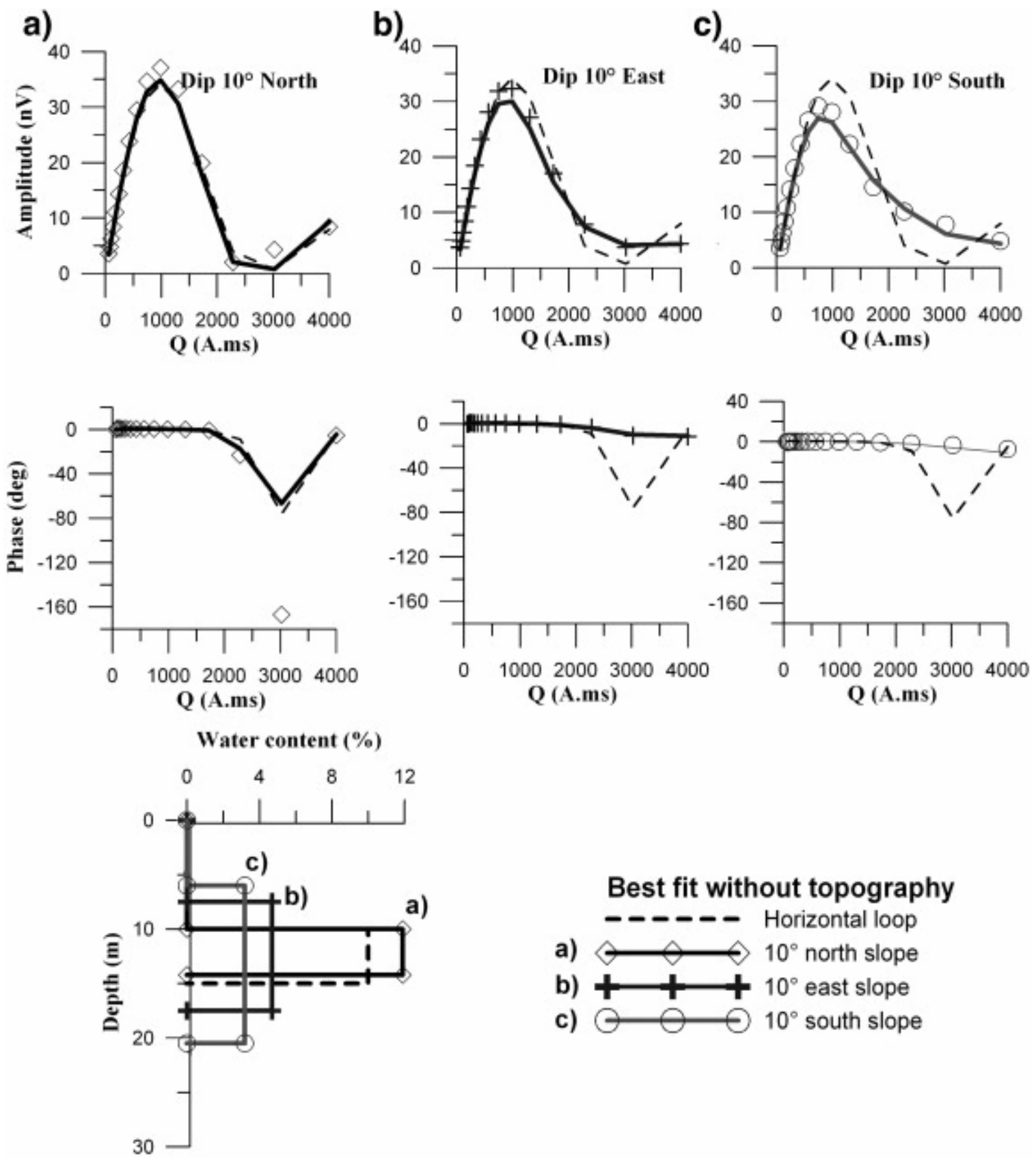

Fig. 8. MRS responses of a 10-15 m aquifer with $10 \%$ water content, using a circular $50 \mathrm{~m}$ diameter ( 1 turn) loop. Surface slope is $10^{\circ}$ and 3 azimuths are considered. For each case, the solid line is the best fit if one neglects the slope, dashed line is the horizontal case. 


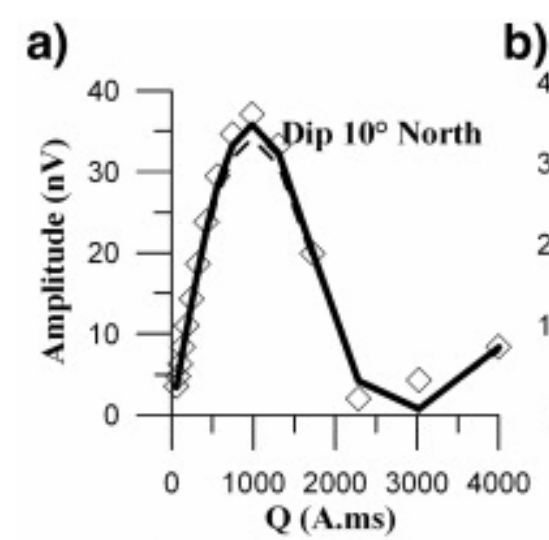

b) c)
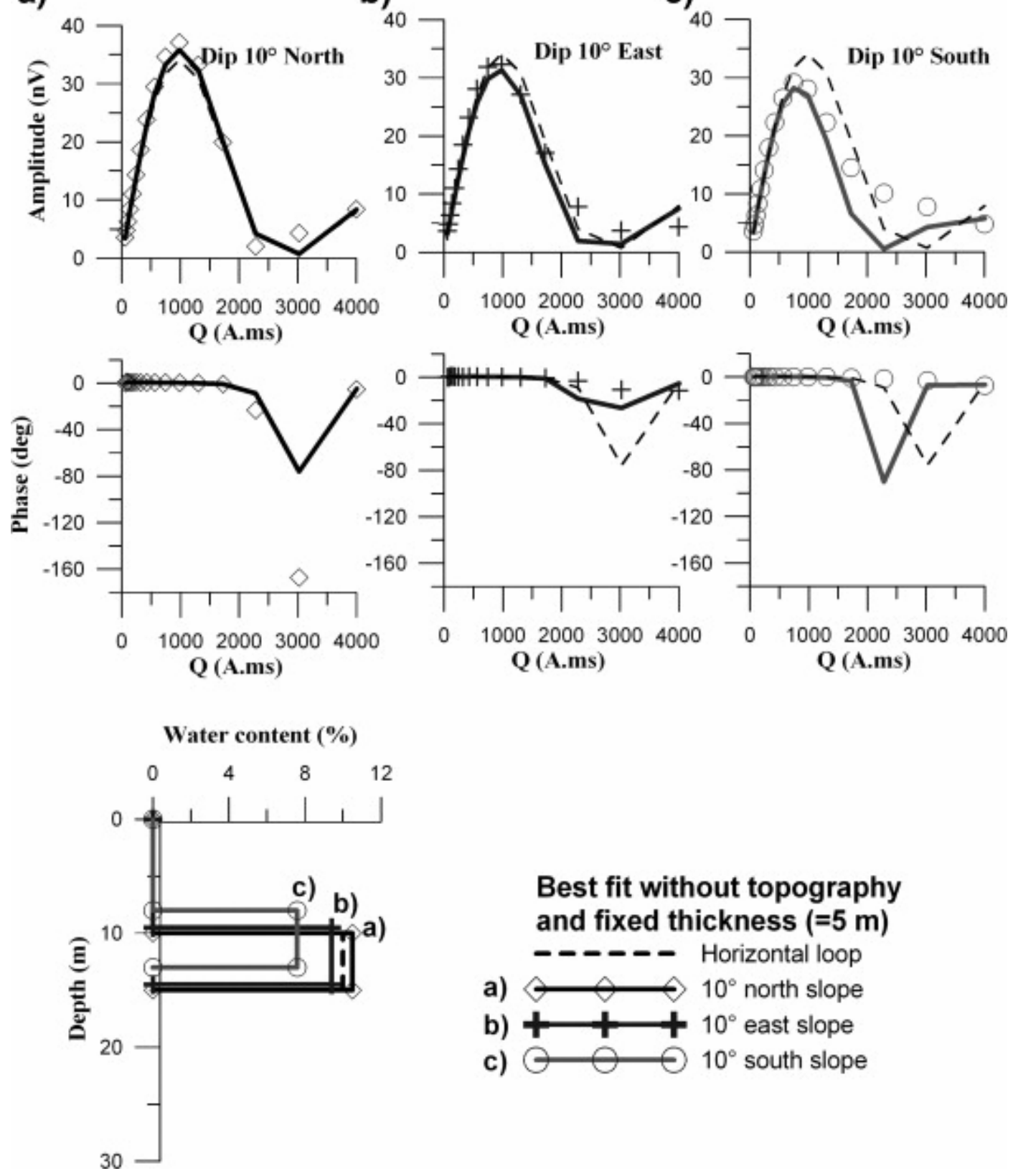

Fig. 9. MRS responses of a 10-15 m aquifer with $10 \%$ water content, using a circular $50 \mathrm{~m}$ diameter ( 1 turn) loop. Surface slope is $10^{\circ}$ and 3 azimuths are considered. For each case, the solid line is the best fit if one neglects the slope but thickness of aquifer is fixed ( $5 \mathrm{~m}$ equal to exact model), dashed line is the horizontal case. 


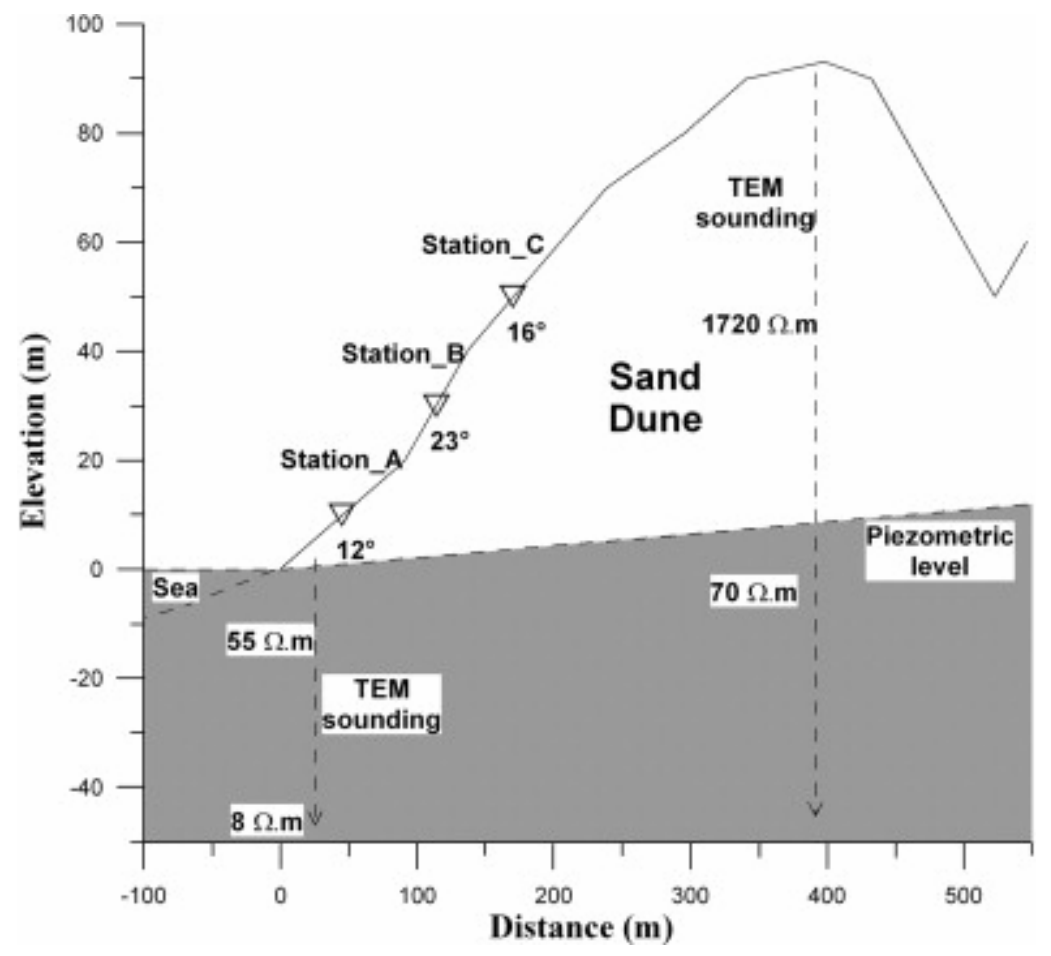

Fig. 10. Pyla sand dune topography (France), with water table extrapolated from a nearby borehole and the sea level, two resistivity logs from TEM and MRS stations. 


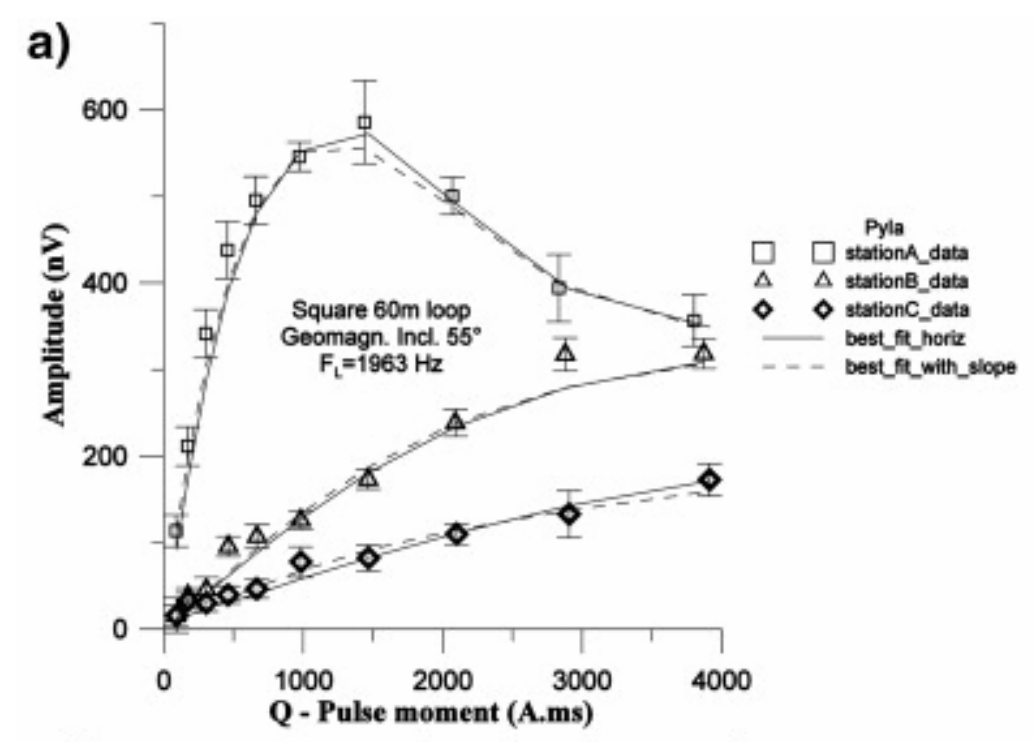

b)

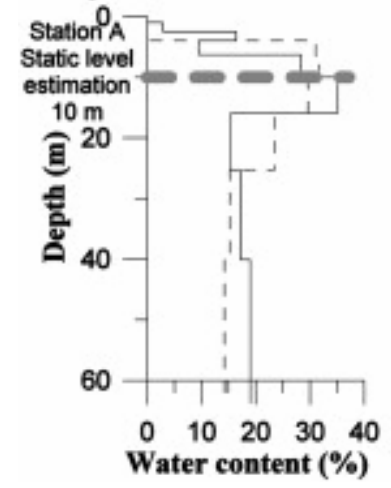

c)

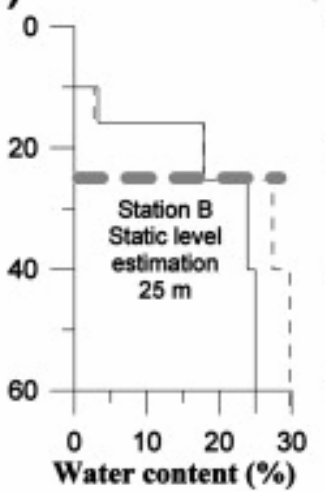

d)

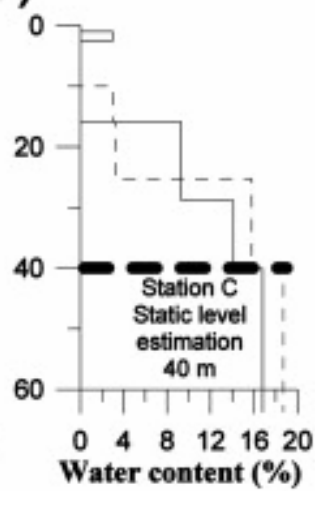

Fig. 11. MRS field data (a), with 60 square loop, performed at three elevations along the Pyla sand dune slope and corresponding inversion results $(b, c, d)$. 
Table 1. / Inversions results of a 5-m-thick, 10\%-water-content, and 10-m-deep layer using a circular $50 \mathrm{~m}$ diameter loop when the slope toward north, east or south is neglected

\begin{tabular}{|c|c|c|c|c|c|c|c|}
\hline \multirow[t]{2}{*}{ Synthetic cases } & \multicolumn{3}{|c|}{ All free parameters } & \multicolumn{3}{|c|}{$\Delta z$ fixed } & \multirow[t]{2}{*}{ Exact model } \\
\hline & South & North & East & South & North & East & \\
\hline Top (m) & 6.0 & 10.0 & 7.5 & 8.0 & 10.0 & 9.5 & 10.0 \\
\hline$\Delta z(\mathrm{~m})$ & 14.5 & 4.2 & 10.0 & 5.0 & 5.0 & 5.0 & 5.0 \\
\hline $\mathrm{Top}+\Delta z / 2(\mathrm{~m})$ & 13.25 & 12.1 & 12.5 & 10.5 & 12.5 & 12 & 12.5 \\
\hline Water content $W(\%)$ & 3.2 & 11.9 & 4.7 & 7.6 & 10.5 & 9.0 & 10.0 \\
\hline$W \cdot \Delta z(\mathrm{~m})$ & 0.46 & 0.50 & 0.47 & 0.38 & 0.52 & 0.45 & 0.50 \\
\hline
\end{tabular}

